\title{
The Chemistry of Photon-Dominated Regions
}

\author{
AMIEL STERNBERG \\ School of Physics and Astronomy, Tel Aviv University, Ramat Aviv, Israel
}

September 5, 1991

Abstract. Theoretical models of photon-dominated regions (PDRs) of molecular cloud surfaces are described. Key aspects of the chemistry of dense PDRs are presented.

\section{Introduction}

The surface layers of the clumpy molecular clouds in star forming regions are commonly exposed to intense fluxes of far-ultraviolet (6-13.6 eV) photons emitted by external or embedded OB stars. The physical and chemical structures of these neutral hydrogen surface layers are controlled by the FUV photons and are therefore called photon-dominated regions (PDRs). Many atomic and molecular emission lines are produced in PDRs. Recent observational results are summarized elsewhere in this volume (see articles by White, Stutzki, and Jaffe). Here I discuss theoretical models of the chemistry of PDRs. Other reviews of PDRs have been presented by Genzel, Harris and Stutzki (1989) and by Hollenbach (1990).

Most of the PDR models in the literature (e.g. De Jong, Boland and Dalgarno 1980, Tielens and Hollenbach 1985, van Dishoeck and Black 1988, Sternberg and Dalgarno 1989) are one dimensional and assume steady state conditions. The models of Tielens and Hollenbach (1985) and Sternberg and Dalgarno (1989) explicitly compute the gas temperature and the gas-phase chemical abundances as functions of cloud depth.

The hydrogen particle density, $n\left(\mathrm{~cm}^{-3}\right)$, and the UV intensity, $\chi$ (in units of the average interstellar FUV intensity) are crucial parameters which govern PDR structure. In star forming regions $n$ ranges from $10^{3}$ to $10^{7}$ $\mathrm{cm}^{-3}$ and $\chi$ ranges from $10^{2}-10^{6}$. In many low density $\left(n<10^{5} \mathrm{~cm}^{-3}\right)$ PDRs the dominant gas heating mechanism in the outer layers is probably dust photoelectric emission. In low density PDRs the cooling is dominated by fine structure emission of atoms and ions (e.g. CII $157 \mu \mathrm{m}$ and OI 63 $\mu \mathrm{m})$. In high density regions $\left(n>10^{5} \mathrm{~cm}^{-3}\right)$ collisional deexcitation of UVpumped $\mathrm{H}_{2}$ molecules becomes an effective heating mechanism. Molecular line cooling $\left(\mathrm{H}_{2}, \mathrm{CO}, \mathrm{OH}\right)$ becomes significant in high density PDRs.

The model calculations show that in dense PDRs the outer atomic hydrogen surface layers become hot $(T>1000 \mathrm{~K})$. The gas temperature falls rapidly in the transition region where the hydrogen becomes molecular. The extent of the hot atomic hydrogen layer depend on the ratio $\chi / n$ (Sternberg and Dalgarno 1989). When $\chi / n>0.01 \mathrm{~cm}^{3}$ the extent of the atomic layer is limited by dust absorption and is of order one visual extinction, $\mathrm{A}_{v}$. When 
$\chi / n<0.01 \mathrm{~cm}^{3}$ the extent of the atomic layer is limited by the opacity of the $H_{2}$ FUV absorption lines and is therefore much smaller. Thus, for fixed $\chi \mathrm{H}_{2}$ self-shielding reduces the sizes of the hot layers as $n$ increases.

\section{Chemistry}

The chemical structure of dense PDRs is illustrated in Fig. 1 (adapted from Sternberg and Dalgarno 1991) which diplays the fractional abundances of various atomic and molecular species as functions of $\mathrm{A}_{v}$ for a cloud with $n=$ $10^{6} \mathrm{~cm}^{-3}$ and $\chi=10^{5}$. The total abundances of carbon and oxygen relative to hydrogen are $3 \times 10^{-4}$ and $6 \times 10^{-4}$. At the cloud surface grain surface $\mathrm{H}_{2}$
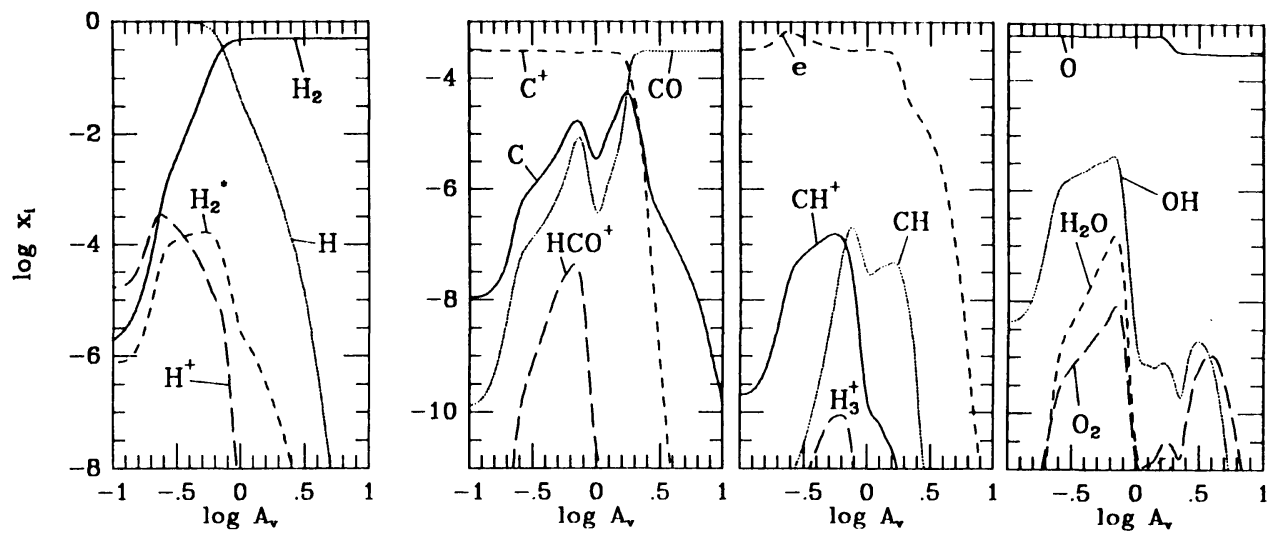

Fig. 1.

formation is slow compared to molecular photodissociation and the hydrogen is atomic. At large cloud depths the FUV radiation is attenuated and the hydrogen becomes molecular. Similarly, at the cloud surface all of the carbon is in the form of $C^{+}$and at large cloud depths it is fully incorporated into $C O$. At intermediate depths the neutral atomic carbon fraction becomes large. Note that the atomic carbon abundance profile has two peaks. At the inner peak $\left(\mathrm{A}_{v}=1.6\right)$ the gas temperature is less than $100 \mathrm{~K}$, the hydrogen is fully molecular, and the carbon chemistry is governed by ion-molecule and neutral reactions which proceed at low temperature. The atomic carbon abundance is set by the balance between photoionization and recombination

$$
C+\nu \leftrightarrow C^{+}+e
$$

$\mathrm{CH}^{+}$and $\mathrm{CH}$ are formed by the slow sequence initiated by radiative association

$$
\begin{aligned}
& C^{+}+\mathrm{H}_{2} \rightarrow \mathrm{CH}_{2}^{+}+\nu \\
& \mathrm{CH}_{2}^{+}+e \rightarrow C H+H
\end{aligned}
$$




$$
\begin{aligned}
C H+\nu & \rightarrow C^{+}+e \\
C H+C^{+} & \rightarrow C^{+}+C
\end{aligned}
$$

$C O$ and $O H$ are formed by the neutral reactions

$$
\begin{aligned}
& C H+O \rightarrow C O+H \\
& C H+O \rightarrow O H+C
\end{aligned}
$$

and are removed by photodissociation.

At the outer atomic carbon peak $\left(A_{v}=0.6\right)$ the gas temperature is greater than $1000 \mathrm{~K}$, the hydrogen is primarily atomic and the chemistry is dominated by reactions with moderate endothermicities or activation energies. Thus, $\mathrm{CH}^{+}$is formed rapidly by the abstraction reaction

$$
\mathrm{C}^{+}+\mathrm{H}_{2} \rightarrow \mathrm{CH}^{+}+\mathrm{H}
$$

which leads to the rapid production of $C H$ via another abstraction reaction

$$
\mathrm{CH}^{+}+\mathrm{H}_{2} \rightarrow \mathrm{CH}_{2}^{+}+\mathrm{H}
$$

followed by reaction (3). The resulting $\mathrm{CH}$ abundance is sufficiently large that the reactions

$$
\begin{aligned}
C H+\nu & \rightarrow C+H \\
C H+H & \rightarrow C+H_{2}
\end{aligned}
$$

dominate the production of atomic carbon rather than reaction (1). A large proton abundance is maintained in the hot gas via the photodissociation reaction

$$
\mathrm{CH}^{+}+\nu \rightarrow C+\mathrm{H}^{+} \text {. }
$$

$\mathrm{OH}$ and $\mathrm{H}_{2} \mathrm{O}$ are formed rapidly via the neutral reactions

$$
\begin{gathered}
\mathrm{O}+\mathrm{H}_{2} \rightarrow \mathrm{OH}+\mathrm{H} \\
\mathrm{OH}+\mathrm{H}_{2} \rightarrow \mathrm{H}_{2} \mathrm{O}+\mathrm{H}
\end{gathered}
$$

which become rapid at high temperatures. $O H$ is a crucial intermediary in hot gas chemistry ( $c f$. Neufeld and Dalgarno 1989) and its large abundance generates the $\mathrm{CO}$ and $\mathrm{HCO}^{+}$and $\mathrm{O}_{2}$ peaks in Fig. 1 via the reactions

$$
\begin{aligned}
\mathrm{C}^{+}+\mathrm{OH} & \rightarrow \mathrm{CO}+\mathrm{H}^{+} \\
\mathrm{C}^{+}+\mathrm{OH} & \rightarrow \mathrm{CO}^{+}+\mathrm{H} \\
\mathrm{CO}^{+}+\mathrm{H}_{2} & \rightarrow \mathrm{HCO}^{+}+\mathrm{H} \\
\mathrm{O}+\mathrm{OH} & \rightarrow \mathrm{O}_{2}+\mathrm{H} .
\end{aligned}
$$


The large $O H$ abundances produced in dense PDRs suggests that they may be the sites of the $O H$ maser spots that surround compact HII regions (Hartquist and Sternberg, 1991).

The molecular abundance peaks that form in the hot regions are sources of intense collisionally excited molecular line emission. Near infrared emission from vibrationally excited hydrogen molecules $\left(H_{2}^{*}\right)$ and far-infrared lines of rotationally excited CO are produced in the hot gas (Sternberg 1986, Hollenbach 1988, Sternberg and Dalgarno 1989, Burton, Hollenbach and Tielens 1990). The double lobed $H_{2}$ emission source recently discovered in the narrow line region of the Seyfert galaxy NGC 1068 may be produced in dense PDRs (Rotaciuc et al. 1991).

\section{Future Work}

The theoretical models have been successful in interpreting and stimulating many observations. Detailed comparison with the observations now require that the models be improved to include the effects of time dependence, clumpy cloud structure, finite cloud size, and non-stellar radiation fields.

\section{Acknowledgements}

This work was supported in part by the Max-Planck Institut für extraterrestrische Phyisk, Garching, Germany.

\section{References}

Burton M., Hollenbach D.J. and Tielens A.G.G.M., 1990, Ap.J. 365,620.

deJong T., Dalgarno A. and Boland W., 1980, Astr.Ap. 91,68.

Genzel R., Harris A.I. and Stutzki J., 1989, in Infrared Spectroscopy in Astronmy ed. M. Kessler (ESA SP series).

Hartquist T.W. and Sternberg A., 1991, M.N.R.A.S. 248,48.

Hollenbach D.J., 1988, Astr. Lett. and Comm. 26, 191.

Hollenbach D.J., 1990 in Evolution of the Interstellar Medium ed. L. Blitz

Neufeld D.A. and Dalgarno A., 1989, Ap. J. 340,869.

Rotatiuc V., Krabbe A., Cameron M., Drapatz S., Genzel R., Sternberg A. and Storey J.W.V., 1991, Ap.J.Letters 370,L23.

Sternberg A., 1986, Ph.D. thesis Columbia University

Sternberg A., 1988, Ap.J. 322,400.

Sternberg A. and Dalgarno A., 1989, Ap.J. 338,197.

Sternberg A. and Dalgarno A., 1991, in prep.

Tielens A.G.G.M. and Hollenbach D.J., 1985, Ap.J. $291,722$.

van Dishoeck E.F. and Black J.H., 1988, Ap.J. 334,711. 